\title{
Steiner trades that give rise to completely decomposable latin interchanges
}

\author{
Richard Bean, Diane Donovan, Abdollah Khodkar and Anne Penfold Street \\ Centre for Discrete Mathematics and Computing \\ Department of Mathematics \\ The University of Queensland, 4072, Australia
}

\begin{abstract}
In this paper we focus on the representation of Steiner trades of volume less than or equal to nine and identify those for which the associated partial latin square can be decomposed into six disjoint latin interchanges.
\end{abstract}

\section{Background information}

In any combinatorial configuration it is possible to identify a subset which uniquely determines the structure of the configuration and in some cases is minimal with respect to this property. For example such subsets can be found by studying the literature on critical sets in latin squares (see Donovan and Howse [2]) and defining sets in block designs (see Street [7]), as well as in the study of premature partial latin squares (see Brankovic, Horak, Miller and Rosa [1]). Research has shown that computer analysis of critical sets, defining sets and premature partial latin squares is computationally expensive. This fact has led to a study of the inherent nature of the configuration in order to obtain information for refining searches and associated algorithms. In the past, critical sets, defining sets and premature partial latin squares have been studied in isolation and, in many cases, using different techniques. However, there is much to be gained by studying these configurations in unison. A crucial element in the identification of defining sets or critical sets is the determination of interchangeable elements within the design or latin square. By representing the interchangeable sets in certain designs as associated partial latin squares, Donovan, Khodkar and Street [3], [4] have identified new families of defining sets. The work in the papers [3], [4] raises many new questions. For instance, can our knowledge of the interchangeable sets in latin squares (latin interchanges) be used to classify the interchangeable sets in the block designs (trades)? It is this interesting question that we focus on here. In Section 2 of this paper we give the appropriate definitions of (partial) latin squares and latin interchanges, (partial) Steiner triple systems and associated Steiner trades, and finally detail the connection between latin interchanges and Steiner trades. In Section 3 we take all Steiner trades of volume less than or equal to nine and classify them according to the structure of the associated latin interchanges. 


\section{Definitions}

First we turn our attention to partial latin squares. A partial latin square $P$ of order $v$ is a $v \times v$ array with entries chosen from the set $V=\{1, \ldots, v\}$ in such a way that each element of $V$ occurs at most once in each row and at most once in each column of the array. For ease of exposition, a partial latin square $P$ will be represented by a set of ordered triples $\left\{\left(i, j ; P_{i j}\right) \mid\right.$ element $P_{i j}$ occurs in cell $(i, j)$ of the array\}. If all the cells of the array are filled then the partial latin square is termed a latin square. That is, a latin square $L$ of order $v$ is a $v \times v$ array with entries chosen from the set $V=\{1, \ldots, v\}$ in such a way that each element of $V$ occurs precisely once in each row and precisely once in each column of the array.

Example 1. The following arrays are examples of three partial latin squares of order 6 . Note that the first may also be termed a latin square.

\begin{tabular}{|c|c|c|c|c|c|}
\hline 1 & 3 & 2 & 4 & 6 & 5 \\
\hline 3 & 2 & 1 & 6 & 5 & 4 \\
\hline 2 & 1 & 3 & 5 & 4 & 6 \\
\hline 4 & 6 & 5 & 1 & 3 & 2 \\
\hline 6 & 5 & 4 & 3 & 2 & 1 \\
\hline 5 & 4 & 6 & 2 & 1 & 3 \\
\hline
\end{tabular}

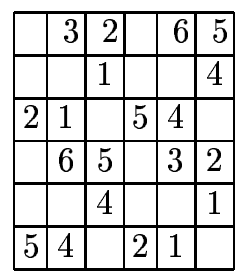

\begin{tabular}{|c|c|c|c|}
\hline & 5 & & \begin{tabular}{l|l}
3 & 2
\end{tabular} \\
\hline & 4 & & 1 \\
\hline $5 \mid 4$ & & 2 & \\
\hline 3 & 2 & & \begin{tabular}{l|l}
6 & 5
\end{tabular} \\
\hline & 1 & & 4 \\
\hline \begin{tabular}{l|l}
2 & 1
\end{tabular} & & & \begin{tabular}{l|l}
4 &
\end{tabular} \\
\hline
\end{tabular}

The set of cells $\mathcal{S}_{P}=\left\{(i, j) \mid\left(i, j ; P_{i j}\right) \in P\right.$, for some $\left.P_{i j} \in V\right\}$ is said to determine the shape of $P$ and $\left|\mathcal{S}_{P}\right|$ is said to be the volume of the partial latin square. That is, the volume is the number of non-empty cells. For each $r$, $1 \leq r \leq v$, let $\mathcal{R}_{P}^{r}$ denote the set of entries occurring in row $r$ of $P$. Formally, $\mathcal{R}_{P}^{r}=\left\{P_{r j} \mid\left(r, j ; P_{r j}\right) \in P\right\}$. Similarly, for each $c, 1 \leq c \leq v$, we define $\mathcal{C}_{P}^{c}=\left\{P_{i c} \mid\left(i, c ; P_{i c}\right) \in P\right\}$ and for each element $e \in V$ we define $\mathcal{E}_{P}^{e}=\{(i, j) \mid$ $(i, j ; e) \in P\}$.

A latin interchange, $\mathcal{I}=\left(I, I^{\prime}\right)$, of volume $s$, is a collection of two partial latin squares, of order $v$, such that

1. $\mathcal{S}_{I}=\mathcal{S}_{I^{\prime}}$,

2. for each $(i, j) \in \mathcal{S}_{I}, I_{i j} \neq I^{\prime}{ }_{i j}$,

3. for each $r, 1 \leq r \leq v, \mathcal{R}_{I}^{r}=\mathcal{R}_{I^{\prime}}^{r}$, and

4. for each $c, 1 \leq c \leq v, \mathcal{C}_{I}^{c}=\mathcal{C}_{I^{\prime}}^{c}$.

Thus a latin interchange is a pair of disjoint partial latin squares of the same shape and order, which are row-wise and column-wise mutually balanced.

Example 2. Together the second and third partial latin square given in Example 1 form a latin interchange of order 6 and of volume 20 .

Let $P$ be a partial Latin square of order $n$ and $\{a, b, c\}=\{1,2,3\}$. Then the $(a, b, c)$-conjugate of $P$ is denoted and defined by

$$
P_{(a, b, c)}=\left\{\left(x_{a}, x_{b} ; x_{c}\right) \mid\left(x_{1}, x_{2} ; x_{3}\right) \in P\right\} .
$$

Note that $P$ has six conjugates. These conjugates are not necessarily distinct. 
For a given latin interchange $\mathcal{I}=\left(I, I^{\prime}\right),\left|\mathcal{R}_{I}^{r}\right|$ denotes the number of nonempty cells in row $r$ and $\left|\mathcal{C}_{I}^{c}\right|$ denotes the number of non-empty cells in column $c$. In addition, $\left|\mathcal{E}_{I}^{e}\right|$ denotes the number of cells which contain element $e \in V$. It is clear that for all $i, j, k \in V,\left|\mathcal{R}_{I}^{i}\right| \geq 2,\left|\mathcal{C}_{I}^{j}\right| \geq 2,\left|\mathcal{E}_{I}^{k}\right| \geq 2$ and $\sum_{i=1}^{v}\left|\mathcal{R}_{I}^{i}\right|=$ $\sum_{j=1}^{v}\left|\mathcal{C}_{I}^{j}\right|=\sum_{k=1}^{v}\left|\mathcal{E}_{I}^{k}\right|=\left|\mathcal{S}_{I}\right|$. In [5], Keedwell introduced the definition of the type of a latin interchange. In this paper we modify this definition slightly and let the type of the latin interchange $\mathcal{I}=\left(I, I^{\prime}\right)$ be

$$
\left(\begin{array}{c}
\left|\mathcal{C}_{I}^{1}\right|+\left|\mathcal{C}_{I}^{2}\right|+\left|\mathcal{C}_{I}^{3}\right|+\ldots+\left|\mathcal{C}_{I}^{v}\right| \\
\left|\mathcal{R}_{I}^{1}\right|+\left|\mathcal{R}_{I}^{2}\right|+\left|\mathcal{R}_{I}^{3}\right|+\ldots+\left|\mathcal{R}_{I}^{v}\right| \\
\left|\mathcal{E}_{I}^{1}\right|+\left|\mathcal{E}_{I}^{2}\right|+\left|\mathcal{E}_{I}^{3}\right|+\ldots+\left|\mathcal{E}_{I}^{v}\right|
\end{array}\right)
$$

Note that if any of the values $\left|\mathcal{C}_{I}^{i}\right|,\left|\mathcal{R}_{I}^{i}\right|$ or $\left|\mathcal{E}_{I}^{i}\right|$ in the above vector are zero, then they are omitted. The type of the latin interchange given in the above example is

$$
\left(\begin{array}{l}
2+4+4+2+4+4 \\
4+2+4+4+2+4 \\
4+4+2+4+4+2
\end{array}\right)
$$

Next we give the definition of a partial Steiner triple system. Let $V=$ $\{1, \ldots, v\}$ and let $\mathcal{B}$ be a collection of 3 -subsets chosen from $V$ in such a way that each 2-subset of $V$ occurs in at most one of the 3 -subsets. Then $(V, \mathcal{B})$ is said to be a partial Steiner triple system and is sometimes referred to as a $2-(v, 3)$ partial Steiner system. The 3-subsets are called blocks or triples and the replication number for a given element $e \in V$ is the number of triples which contain $e$. If $|\mathcal{B}|=v(v-1) / 6$ then each of the 2-subsets of $V$ is contained in precisely one triple of $\mathcal{B}$ and in this case $(V, \mathcal{B})$ is said to be a Steiner triple system of order $v$.

Take two such partial Steiner triple systems with triples $T$ and $T^{\prime}$. If $|T|=$ $\left|T^{\prime}\right|$ and each of the 2-subsets of elements of $V$ contained in the triples of $T$ are also contained in the triples of $T^{\prime}$, then $T$ and $T^{\prime}$ are said to be mutually balanced. If $T$ and $T^{\prime}$ are mutually balanced and have no common triples, they form a 2$(v, 3)$ Steiner trade usually denoted by $\mathcal{T}=\left(T, T^{\prime}\right)$. The volume $(T)$ of the trade is $|T|$ and the foundation of $\mathcal{T}$ is $F(\mathcal{T})=\{x \mid x$ is contained in a triple of $T\}$.

Let $(V, \mathcal{B})$ be a partial Steiner triple system of order $v$. We define the corresponding partial Steiner latin square of order $v$ to be the array with entry $k$ in cell $(i, j)$ if and only if $\{i, j, k\} \in \mathcal{B}$. We emphasise that for each triple $\{x, y, z\} \in T\left(\{u, v, w\} \in T^{\prime}\right), I$ contains six entries $(x, y ; z),(x, z ; y),(y, x ; z)$, $(y, z ; x),(z, y ; x),(z, x ; y)\left(I^{\prime}\right.$ contains six entries $(u, v ; w),(u, w ; v),(v, u ; w)$, $(v, w ; u),(w, v ; u),(w, u ; v))$.

Lemma 1. Let $\mathcal{T}=\left(T, T^{\prime}\right)$ be a 2-(v,3) Steiner trade. Then the partial Steiner latin squares $I$ and $I^{\prime}$ corresponding to $T$ and $T^{\prime}$, respectively, form a latin interchange $\mathcal{I}=\left(I, I^{\prime}\right)$.

Proof: Note that $|T|=\left|T^{\prime}\right|$ and $T \cap T^{\prime}=\emptyset$, hence $I$ and $I^{\prime}$ have the same volume and shape and are disjoint. Next assume that the rows of $I$ and $I^{\prime}$ 
are not mutually balanced. That is, for some row $r$ there exists a column $j$ such that $(r, j ; z) \in I$, but for the same row $r\left(r, j^{\prime} ; z\right) \notin I^{\prime}$ for any column $j^{\prime}$. Correspondingly the triple $\{r, j, z\} \in T$ for some $j \in V$, but $\left\{r, j^{\prime}, z\right\} \notin T^{\prime}$ for any $j^{\prime} \in V$, which is a contradiction as $\mathcal{T}=\left(T, T^{\prime}\right)$ is a trade. We may obtain a similar contradiction for the columns and so deduce that the rows and columns of $I$ and $I^{\prime}$ are mutually balanced. Consequently $\mathcal{I}=\left(I, I^{\prime}\right)$ is a latin interchange as required.

In [4] Donovan, Khodkar and Street showed that for the given trade $\mathcal{T}=$ $\left(T, T^{\prime}\right)$, where $T=\{123,145,167,248,368,578\}$ and $T^{\prime}=\{124,136,157,238$, $458,678\}$, the partial latin interchange $\mathcal{I}$ corresponding to the triples of $\mathcal{T}$ can be decomposed into six disjoint latin interchanges, $\mathcal{I}_{i}=\left(I_{i}, I_{i}^{\prime}\right)$ for $i=1, \ldots, 6$, in such a way that for each $i$ there is a one-to-one correspondence between the entries of $I_{i}$ and the triples of $T$. Further, they showed that no such decomposition exists for the latin interchange associated with the trade $\mathcal{T}=\left(T, T^{\prime}\right)$, where $T=\{123,145,167,247,346,357\}$ and $T^{\prime}=\{124,136,157,237,345,467\}$. These results raise the following question:

QUESTION 1: For which trades $\mathcal{T}=\left(T, T^{\prime}\right)$ can the corresponding latin interchange $\mathcal{I}=\left(I, I^{\prime}\right)$ be decomposed into six disjoint latin interchanges $\mathcal{I}_{i}=$ $\left(I_{i}, I_{i}^{\prime}\right), 1 \leq i \leq 6$, such that for $i=1, \ldots, 6$ there is a one-to-one correspondence between the triples of $T\left(T^{\prime}\right)$ and the entries of $I_{i}\left(I_{i}^{\prime}\right)$ which maps $\{x, y, z\} \in T$ to $(x, y ; z) \in I_{i}$ ?

In this paper we give some partial answers to this question and, in particular, give an exact answer for all Steiner trades with block size three and volume less than or equal to nine. Our list of trades of volume less than or equal to nine has been taken from [6] where Khosrovshahi and Maimani completely classified all Steiner trades with block size three and volumes six to nine.

\section{Partial Answers}

We begin by stating a result which identifies some Steiner trades whose corresponding partial Steiner latin squares can be decomposed into six disjoint latin interchanges. First we need a definition.

Let $\mathcal{T}=\left(T, T^{\prime}\right)$ be a trade. We say $\mathcal{T}$ is a minimal trade if there is no $\emptyset \neq B \subset T$ and $\emptyset \neq B^{\prime} \subset T^{\prime}$ such that $\left(T \backslash B, T^{\prime} \backslash B^{\prime}\right)$ is a trade.

Lemma 2. Let $\mathcal{T}=\left(T, T^{\prime}\right)$ be a Steiner minimal trade. For each element $x \in$ $F(\mathcal{T})$ let there exist a subset $S_{x}$ of $F(\mathcal{T})$ such that $x \in S_{x}$ and each triple of $T$ intersects the set $S_{x}$ in precisely one element. Then the latin interchange corresponding to $\mathcal{T}=\left(T, T^{\prime}\right)$, say $\mathcal{I}=\left(I, I^{\prime}\right)$, can be decomposed into six disjoint latin interchanges. 
Proof: First we prove that for $x, y \in F(\mathcal{T})$ we have either $S_{x}=S_{y}$ or $S_{x} \cap S_{y}=\emptyset$. Let $S_{x} \neq S_{y}$ and $S_{x} \cap S_{y} \neq \emptyset$. Define

$$
\begin{aligned}
& T_{1}=\left\{\{a, b, c\} \in T \mid a \in S_{x} \backslash S_{y}, b \in S_{y} \backslash S_{x}, c \in F(\mathcal{T}) \backslash\left(S_{x} \cup S_{y}\right)\right\}, \\
& T_{2}=\left\{\{d, e, f\} \in T \mid d \in S_{x} \cap S_{y}, e, f \in F(\mathcal{T}) \backslash\left(S_{x} \cup S_{y}\right)\right\} \\
& T_{1}^{\prime}=\left\{\left\{a^{\prime}, b^{\prime}, c^{\prime}\right\} \in T^{\prime} \mid a^{\prime} \in S_{x} \backslash S_{y}, b^{\prime} \in S_{y} \backslash S_{x}, c^{\prime} \in F(\mathcal{T}) \backslash\left(S_{x} \cup S_{y}\right)\right\} \text { and } \\
& T_{2}^{\prime}=\left\{\left\{d^{\prime}, e^{\prime}, f^{\prime}\right\} \in T^{\prime} \mid d^{\prime} \in S_{x} \cap S_{y}, e^{\prime}, f^{\prime} \in F(\mathcal{T}) \backslash\left(S_{x} \cup S_{y}\right)\right\}
\end{aligned}
$$

We note that if the pair $\{a, b\}$ occurs in a triple of $T$ then $a$ and $b$ cannot both be in $S_{z}$ for any $z \in \mathcal{T}$. This leads to $T=T_{1} \cup T_{2}$ and $T^{\prime}=T_{1}^{\prime} \cup T_{2}^{\prime}$. Now if the pair $\{a, b\}$ is in a triple of $T_{1}$ then $\{a, b\}$ is in a triple of $T_{1}^{\prime}$. So $\left(T_{1}, T_{1}^{\prime}\right)$ is a Steiner trade. This is a contradiction since $\mathcal{T}=\left(T, T^{\prime}\right)$ is minimal. Hence either $S_{x}=S_{y}$ or $S_{x} \cap S_{y}=\emptyset$ for $x, y \in F(\mathcal{T})$.

Now let the triple $\{a, b, c\}$ be in $T$; then $S_{a} \cup S_{b} \cup S_{c}=F(\mathcal{T}), S_{a} \cap S_{b}=\emptyset$, $S_{a} \cap S_{c}=\emptyset$ and $S_{b} \cap S_{c}=\emptyset$. We define

$$
I_{1}=\left\{(x, y ; z) \mid\{x, y, z\} \in T, x \in S_{a}, y \in S_{b}, z \in S_{c}\right\} .
$$

It is easy to see that there is a one-to-one correspondence between the elements of $I_{1}$ and the triples of $T$. Moreover, $I_{1}$ forms half of an interchange into which we are decomposing the partial latin square associated with $T$. We leave the reader to prove that the six conjugates of $I_{1}$ decompose $I$ into six disjoint latin interchanges, where $\mathcal{I}=\left(I, I^{\prime}\right)$ is the latin interchange corresponding to $\mathcal{T}=$ $\left(T, T^{\prime}\right)$.

However, the above condition is not necessary as is shown by the following example.

Example 3. The partial Steiner latin square corresponding to the trade $\mathcal{T}=$ $\left(T, T^{\prime}\right)$ where $T=\{136,148,159,239,246,257,347,358\}$ and $T^{\prime}=\{139,158,146$, $259,236,247,357,348\}$ can be decomposed into six disjoint latin interchanges. These may be obtained by taking the conjugates of the latin interchange, $\mathcal{I}_{1}=$ $\left(I_{1}, I_{1}^{\prime}\right)$, where $I_{1}=\{(1,3 ; 6),(1,4 ; 8),(1,5 ; 9),(2,3 ; 9),(2,4 ; 6),(2,5 ; 7),(3,4 ; 7)$, $(3,5 ; 8)\}$. This trade does not have the property set out in the above lemma but is decomposable.

Thus to further our study we focussed on the trades of volume less than or equal to nine and began by developing the following algorithm which we used to systematically test for the decomposition of these Steiner trades.

\section{Algorithm:}

- Input the trade $\mathcal{T}$

- Calculate $|\mathcal{T}|$.

- Convert the trade $\mathcal{T}$ to the associated partial Steiner latin square $P$.

- Generate all $\left\lfloor\frac{|\mathcal{T}|}{2} \times \frac{\lfloor\mathcal{T}\rfloor}{2}\right\rfloor$ subarrays of $P$.

- For each subarray $S$ generate all subsets $U$ of size $|\mathcal{T}|$. 
- Calculate all permutations of size $x$ of the symbols $\{1,2 \ldots, x\}$ with no fixed points, where $2 \leq x \leq\left\lfloor\frac{\mid \mathcal{T}\rfloor}{2}\right\rfloor$.

- Determine the size of each row and column in the subset $U$, and the number of times each element occurs in the subset $U$. If each of these numbers is greater than or equal to 2, continue; else move to the next subset.

- Apply each of the permutations calculated above to each of the rows in each subset $U$. If the columns are mutually balanced then a latin interchange has been found.

- Once all latin interchanges have been determined, check for a one-to-one correspondence between the elements of each latin interchange and the triples of the trade $\mathcal{T}$. If a correspondence is found, then a decomposition of the partial Steiner latin square associated with $\mathcal{T}$ is possible.

There are 25 Steiner trades of volume less than or equal to nine and classifying these further we see that up to isomorphism there is one Steiner trade of volume 4 , two of volume 6 , two of volume 7 , nine of volume 8 and eleven of volume 9 . The triples of these trades are listed below. Our testing verified that for thirteen of these Steiner trades the corresponding partial Steiner latin square can be decomposed into six disjoint latin interchanges satisfying the properties given in Question 1 of Section 2. These thirteen cases are discussed below and the general nature of the decomposition is given. For the remainder of the cases, we present theoretical arguments that indicate why such a decomposition is not possible.

REMARK: We note that if such a decomposition exists for each $i=1, \ldots, 6$ and each $x \in V$, the partial latin square $I_{i}$ is such that $\left|\mathcal{R}_{I_{i}}^{x}\right|+\left|\mathcal{C}_{I_{i}}^{x}\right|+\left|\mathcal{E}_{I_{i}}^{x}\right|$ equals the replication number for element $x$. Also since for $i=1, \ldots, 6,|T|=\left|I_{i}\right|$, the volume of each of the latin interchanges $\mathcal{I}_{i}$ is less than or equal to nine. In the paper [5] Keedwell classified the type of all latin interchanges of volume less than or equal to 10 . We have used his classifications when arguing that decomposition is not possible and in many of these cases we will use the following lemma frequently.

Lemma 3. If the replication number of a symbol e is 2 or 3 , then for $i=1, \ldots, 6$ in any given interchange $\mathcal{I}_{i}$, e can only occur as a row or a column or an element. If the replication number of a symbol $e$ is 4 , then in any given interchange $\mathcal{I}_{i}$, $e$ can only occur as a row, a column, or an element, or a row and a column, a row and an element, or a column and an element.

Proof: Since an interchange requires that $\left|\mathcal{R}_{I}^{e}\right| \geq 2$ and $\left|\mathcal{C}_{I}^{e}\right| \geq 2$, and $\left|\mathcal{E}_{I}^{e}\right| \geq 2$, when the replication number of $e$ is 2 or 3 , the symbol cannot be split between rows and columns, or rows and elements, or columns and elements. Similarly when the replication number of $e$ is 4 , the symbol cannot be split between rows, columns, and elements.

Trade of volume $4 \mathcal{T}_{0}=\left(T, T^{\prime}\right)$ where $T=\{123,156,435,426\}$ and $T^{\prime}=$ $\{126,135,423,456\}$. This trade can be decomposed into interchanges, one of 
which is given by $\mathcal{I}_{1}=\left(I_{1}, I_{1}^{\prime}\right)$ where $I_{1}=\{(2,3 ; 1),(5,6 ; 1),(5,3 ; 4),(2,6 ; 4)\}$. Note that both $T$ and $T^{\prime}$ are Pasch configurations and the trade of volume 4 is nothing else than a "Pasch switch".

Trade of volume $6 \mathcal{T}_{1}=\left(T, T^{\prime}\right)$ where $T=\{123,145,167,247,346,357\}$ and $T^{\prime}=\{124,136,157,237,345,467\}$. The replication numbers for the elements $1, \ldots, 7$ are:

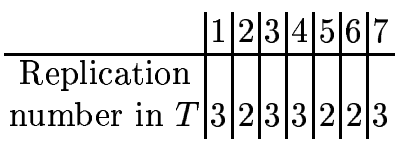

Assume that the latin interchange $\mathcal{I}$ associated with $\mathcal{T}_{1}$ can be decomposed into six disjoint latin interchanges, then since volume $(T)=6$ one of these latin interchanges must have type

$$
\left(\begin{array}{c}
3+3 \\
3+3 \\
2+2+2
\end{array}\right)
$$

So without loss of generality assume column 1 contains three entries, but this implies there are three nonempty rows which is a contradiction. Therefore no such decomposition exists.

Trade of volume $6 \mathcal{T}_{2}=\left(T, T^{\prime}\right)$ where $T=\{123,145,167,248,368,578\}$ and $T^{\prime}=\{124,136,157,238,458,678\}$. This trade can be decomposed into interchanges, one of which is given by $\mathcal{I}_{1}=\left(I_{1}, I_{1}^{\prime}\right)$ where $I_{1}=\{(1,3 ; 2),(1,4 ; 5)$, $(1,7 ; 6),(8,4 ; 2),(8,3 ; 6),(8,7 ; 5)\}$.

Example 4. Here we digress for a moment and use this trade to illustrate Lemma 2. To see this note that $S_{1}=S_{8}=\{1,8\}, S_{2}=S_{5}=S_{6}=\{2,5,6\}$ and $S_{3}=S_{4}=S_{7}=\{3,4,7\}$.

Trade of volume $7 \mathcal{T}_{3}=\left(T, T^{\prime}\right)$ where $T=\{123,145,167,246,257,356,347\}$ and $T^{\prime}=\{124,136,157,237,256,345,467\}$. The only possible type of a latin interchange $\mathcal{I}$ of volume seven is

$$
\left(\begin{array}{c}
3+2+2 \\
3+2+2 \\
3+2+2
\end{array}\right) .
$$

Since the replication number of each element is 3 this type is not possible.

Trade of volume $7 \mathcal{T}_{4}=\left(T, T^{\prime}\right)$ where $T=\{123,145,167,248,358,369,579\}$ and $T^{\prime}=\{124,136,157,238,359,458,679\}$. A decomposition exists in which one interchange $\mathcal{I}_{1}=\left(I_{1}, I_{1}^{\prime}\right)$ is

$$
I=\{(1,2 ; 3),(1,5 ; 4),(1,6 ; 7),(8,2 ; 4),(8,5 ; 3),(9,6 ; 3),(9,5 ; 7)\} .
$$


Trade of volume $8 \mathcal{T}_{5}=\left(T, T^{\prime}\right)$ where $T=\{123,145,167,248,257,346,378$, $568\}$ and $T^{\prime}=\{124,136,157,237,258,348,456,678\}$. As in the trade $\mathcal{T}_{3}$, the replication number for each element is 3 , and so it is not possible to find a type

$$
\left(\begin{array}{c}
W \\
X \\
Y
\end{array}\right)
$$

in which the sums $\mathrm{W}, \mathrm{X}$, and $\mathrm{Y}$ consist only of 3s. Thus no decomposition exists.

Trade of volume $8 \mathcal{T}_{6}=\left(T, T^{\prime}\right)$ where $T=\{123,145,167,246,257,359,368$, $489\}$ and $T^{\prime}=\{124,136,157,235,267,389,459,468\}$. The replication numbers for the elements $1, \ldots, 9$ are as follows.

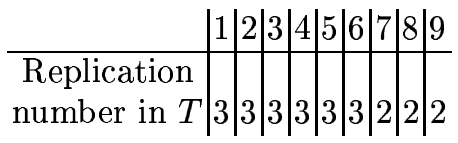

But there is no interchange of size 8 which has type

$$
\left(\begin{array}{c}
3+3+2 \\
3+3+2 \\
3+3+2
\end{array}\right)
$$

and thus no decomposition exists.

Trade of volume $8 \mathcal{T}_{7}=\left(T, T^{\prime}\right)$ where $T=\{123,145,167,189,247,346,358$, $379\}$ and $T^{\prime}=\{124,136,158,179,237,345,389,467\}$. The replication numbers for the elements $1, \ldots, 9$ are as follows.

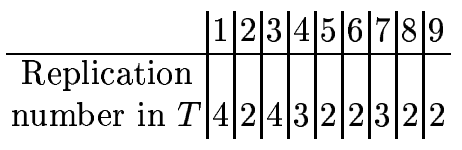

Assume that the latin interchange $\mathcal{I}$ associated with $\mathcal{T}_{7}$ can be decomposed into six disjoint latin interchanges, then since volume $(T)=8$ one of these latin interchanges, $\mathcal{I}_{1}=\left(I_{1}, I_{1}^{\prime}\right)$ say, must have type

$$
\left(\begin{array}{c}
3+3+2 \\
X \\
Y
\end{array}\right)
$$

where $X$ and $Y$ represent the appropriate sum values of $\left|\mathcal{R}_{I_{1}}^{r}\right|$ and $\left|\mathcal{E}_{I_{1}}^{e}\right|$. By Lemma 3 , this implies that both row 4 and row 7 are simultaneously non-empty. Moreover, the elements 4 and 7 cannot occur as entries. This is a contradiction as $247 \in T$. Therefore no such decomposition exists. 
Trade of volume $8 \mathcal{T}_{8}=\left(T, T^{\prime}\right)$ where $T=\{127,138,28 A, 379,459,46 A, 57 A$, $689\}$ and $T^{\prime}=\{128,137,27 A, 389,45 A, 469,579,68 A\}$. A decomposition exists where one interchange is given by $\mathcal{I}_{1}=\left(I_{1}, I_{1}^{\prime}\right)$ where $I_{1}=\{(1,2 ; 7),(1,3 ; 8)$, $(A, 2 ; 8),(9,3 ; 7),(9,5 ; 4),(A, 6 ; 4),(A, 5 ; 7),(9,6 ; 8)\}$.

Trade of volume $8 \mathcal{T}_{9}=\left(T, T^{\prime}\right)$ where $T=\{123,145,167,189,24 A, 268,279$, $35 A\}$ and $T^{\prime}=\{124,135,168,179,23 A, 267,289,45 A\}$. A decomposition exists where one interchange is given by $\mathcal{I}_{1}=\left(I_{1}, I_{1}^{\prime}\right)$ where $I_{1}=\{(1,2 ; 3),(1,5 ; 4)$, $(1,6 ; 7),(1,9 ; 8),(A, 2 ; 4),(2,6 ; 8),(2,9 ; 7),(A, 5 ; 3)\}$.

Trade of volume $8 \mathcal{T}_{10}=\left(T, T^{\prime}\right)$ where $T=\{123,145,167,189,24 A, 35 A, 68 A$, $79 A\}$ and $T^{\prime}=\{124,135,168,179,23 A, 45 A, 67 A, 89 A\}$. A decomposition exists where one interchange is given by $\mathcal{I}_{1}=\left(I_{1}, I_{1}^{\prime}\right)$ where $I_{1}=\{(1,2 ; 3),(1,5 ; 4)$, $(1,6 ; 7),(1,9 ; 8),(A, 2 ; 4),(A, 5 ; 3),(A, 6 ; 8),(A, 9 ; 7)\}$.

Trade of volume $8 \mathcal{T}_{11}=\left(T, T^{\prime}\right)$ where $T=\{123,145,167,189,24 A, 36 A, 58 A$, $79 A\}$ and $T^{\prime}=\{124,136,158,179,23 A, 45 A, 67 A, 89 A\}$. A decomposition exists where one interchange is given by $\mathcal{I}_{1}=\left(I_{1}, I_{1}^{\prime}\right)$ where $I_{1}=\{(1,2 ; 3),(1,5 ; 4)$, $(1,6 ; 7),(1,9 ; 8),(A, 2 ; 4),(A, 6 ; 3),(A, 5 ; 8),(A, 9 ; 7)\}$.

Trade of volume $8 \mathcal{T}_{12}=\left(T, T^{\prime}\right)$ where $T=\{123,145,167,189,24 A, 35 A 68 B$, $79 B\}$ and $T^{\prime}=\{124,135,168,179,23 A, 45 A, 67 B, 89 B\}$. A decomposition exists where one interchange is given by $\mathcal{I}_{1}=\left(I_{1}, I_{1}^{\prime}\right)$ where $I_{1}=\{(3,2 ; 1),(4,5 ; 1)$, $(7,6 ; 1),(8,9 ; 1),(4,2 ; A),(8,6 ; B),(7,9 ; B),(3,5 ; A)\}$.

Trade of volume $8 \mathcal{T}_{13}=\left(T, T^{\prime}\right)$ where $T=\{123,145,24 A, 35 A, 678,69 B, 79 C$, $8 B C\}$ and $T^{\prime}=\{124,135,23 A, 45 A, 67 A, 68 B, 78 C, 9 B C\}$. A decomposition exists where one interchange is given by $\mathcal{I}_{1}=\left(I_{1}, I_{1}^{\prime}\right)$ where $I_{1}=\{(3,2 ; 1),(4,5 ; 1)$, $(4,2 ; A),(3,5 ; A),(8,7 ; 6),(9, B ; 6),(9,7 ; C),(8, B ; C)\}$.

Trade of volume $9 \mathcal{T}_{14}=\left(T, T^{\prime}\right)$ where $T=\{145,167,189,239,257,268,346$, $358,479\}$ and $T^{\prime}=\{146,158,179,235,267,289,349,368,457\}$.

Again the replication number for each element $e$ is 3. By Lemma 3, any latin interchange $I_{1}$ must be a $3 \times 3$ subsquare. Assume that $\mathcal{I}_{1}=\left(I_{1}, I_{1}^{\prime}\right)$ is one of the interchanges into which the partial latin square associated with $\mathcal{T}_{14}$ can be decomposed. There are no $3 \times 3$ subsquares in the partial latin square associated with $T$. We can show this by considering the partial latin square $I_{1}$ containing the element $(4,5 ; 1)$. By Lemma 3,4 can only occur as a row, and 1 can only occur as an element. Because 671 is a triple, 6 must occur only as a row or column. Assume that 6 occurs only as a row. In this case, because 346 is a triple, either $(6,4 ; 3)$ or $(6,3 ; 4)$ must occur in $I_{1}$ which is a contradiction since 4 can only be a row. Thus 6 must occur only as a column. In this case $(7,6 ; 1)$ must be in $I_{1}$ and thus because 479 is a triple, $(7,9 ; 4)$ or $(7,4 ; 9)$ must be an 
element in $I_{1}$ which is a contradiction since we are assuming that 4 is a row. Thus no such decomposition exists.

Trade of volume $9 \mathcal{T}_{15}=\left(T, T^{\prime}\right)$ where $T=\{147,158,169,248,259,267,349$, $357,368\}$ and $T^{\prime}=\{148,159,167,249,257,268,347,358,369\}$. A decomposition exists where one interchange is given by $\mathcal{I}_{1}=\left(I_{1}, I_{1}^{\prime}\right)$ where $I_{1}=\{(1,4 ; 7)$, $(1,5 ; 8),(1,6 ; 9),(2,4 ; 8),(2,5 ; 9),(2,6 ; 7),(3,4 ; 9),(3,5 ; 7),(3,6 ; 8)\}$.

Trade of volume $9 \mathcal{T}_{16}=\left(T, T^{\prime}\right)$ where $T=\{123,145,167,189,248,257,269$, $346,479\}$ and $T^{\prime}=\{125,136,148,179,234,267,289,457,469\}$.

The replication numbers for the elements $1, \ldots, 9$ are as follows.

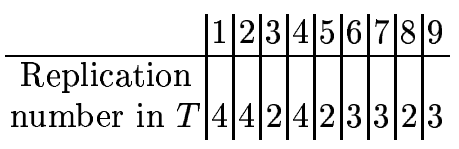

Assume $(6,9 ; 2) \in I_{1}$, where $I_{1}$ forms half of an interchange into which we are decomposing the partial latin square associated with $T$. Then by Lemma 3 we can say that 6 occurs only as a row, and 9 occurs only as a column. Since 167 is a triple, then 7 occurs only as a column or element, and since 479 is a triple, then 7 occurs only as a row or element. This means that 7 occurs only as an element. Thus $\{(6,1 ; 7),(4,9 ; 7)\} \subseteq I_{1}$. With this information, plus the fact that 257 and 145 are triples, we have four cases:

Case $1\{(5,2 ; 7),(5,1 ; 4)\} \subseteq I_{1}$. Since $(6,9 ; 2),(6,1 ; 7)$ and $(4,9 ; 7)$ are also in $I_{1}$ by Lemma 3 we find that $(6,3 ; 4),(1,3 ; 2),(1,9 ; 8)$ and $(4,2 ; 8)$ must be in $I_{1}$. Now it is easy to see that $I_{1}$ is not a latin interchange. This is a contradiction.

Case $2\{(5,2 ; 7),(5,4 ; 1)\} \subseteq I_{1}$. Since $(6,9 ; 2),(6,1 ; 7)$ and $(4,9 ; 7)$ are also in $I_{1}$ by Lemma 3 we find that $(6,4 ; 3) \in I_{1}$. Now either $(1,2 ; 3)$ or $(2,1 ; 3)$ must be in $I_{1}$. But both are impossible by Lemma 3 .

Case $3\{(2,5 ; 7),(4,5 ; 1)\} \subseteq I_{1}$. Since $(6,9 ; 2),(6,1 ; 7)$ and $(4,9 ; 7)$ are also in $I_{1}$ by Lemma 3 we find that $(8,9 ; 1),(8,4 ; 2),(6,4 ; 3)$ and $(2,1 ; 3)$ must be in $I_{1}$. Now it is easy to see that $I_{1}$ with these entries cannot be a latin interchange. This is a contradiction.

Case $4\{(2,5 ; 7),(1,5 ; 4)\} \subseteq I_{1}$. Since $(6,9 ; 2),(6,1 ; 7)$ and $(4,9 ; 7)$ are also in $I_{1}$ by Lemma 3 we find that $(1,9 ; 8) \in I_{1}$. Now either $(4,2 ; 8)$ or $(2,4 ; 8)$ must be in $T_{1}$. But both are impossible by Lemma 3 .

Thus no decomposition exists.

Trade of volume $9 \mathcal{T}_{17}=\left(T, T^{\prime}\right)$ where $T=\{123,145,167,189,248,256,279$, $346,358\}$ and $T^{\prime}=\{124,136,158,179,235,267,289,348,456\}$.

The replication numbers for the elements $1, \ldots, 9$ are as follows.

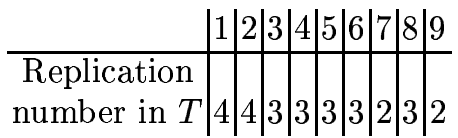


Assume $(3,4 ; 6) \in I_{1}$, where $I_{1}$ forms half of an interchange into which we are decomposing the partial latin square associated with $T$. Then by Lemma 3 we can say that 3 occurs only as a row, 4 occurs only as a column, and 6 occurs only as an element. Since 256 is a triple, then 5 occurs only as a column or a row, and since 358 is a triple, then 5 occurs only as a column or an element. This implies that 5 occurs only as a column. However, this leads to a contradiction since if we look at the triple 145, 4 and 5 must both occur as columns. Thus no decomposition exists.

Trade of volume $9 \mathcal{T}_{18}=\left(T, T^{\prime}\right)$ where $T=\{123,145,167,248,369,378,49 A$, $579,68 A\}$ and $T^{\prime}=\{124,136,157,238,379,459,48 A, 678,69 A\}$.

The replication numbers for the elements $1, \ldots, 9, A$ are as follows.

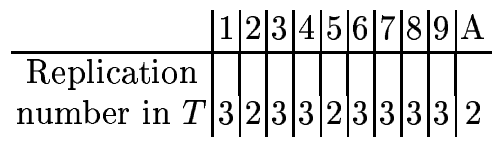

Assume $(1,2 ; 3) \in I_{1}$, where $I_{1}$ forms half of an interchange into which we are decomposing the partial latin square associated with $T$. Then by Lemma 3 we can say that 1 occurs only as a row, 2 occurs only as a column, and 3 occurs only as an element. Since 248 is a triple, we have that 4 occurs only as a row or an element, and since 145 is a triple, we have that 4 occurs only as a column or an element. Therefore, 4 occurs only as an element, 5 occurs only as a column, and 8 occurs only as a row. Since $49 \mathrm{~A}$ is a triple, we have that 9 occurs only as a row or a column, and since 579 is a triple, we have that 9 occurs only as a row or an element. Therefore, 9 occurs only as a row, A occurs only as a column, and 7 occurs only as an element.

However this leads to a contradiction since in the triple 378,3 and 7 must both be elements. Therefore no decomposition exists.

Trade of volume $9 \mathcal{T}_{19}=\left(T, T^{\prime}\right)$ where $T=\{123,145,167,189,24 A, 356,37 A$, $468,479\}$ and $T^{\prime}=\{124,135,168,179,23 A, 367,456,489,47 A\}$. A decomposition exists where one interchange is given by $\mathcal{I}_{1}=\left(I_{1}, I_{1}^{\prime}\right)$ where $I_{1}=\{(1,2 ; 3)$, $(1,5 ; 4),(6,7 ; 1),(9,8 ; 1),(A, 2 ; 4),(6,5 ; 3),(A, 7 ; 3),(6,8 ; 4),(9,7 ; 4)\}$.

Trade of volume $9 \mathcal{T}_{20}=\left(T, T^{\prime}\right)$ where $T=\{123,145,167,189,24 A, 368,39 A$, $479,578\}$ and $T^{\prime}=\{124,136,158,179,23 A, 389,457,49 A, 678\}$.

The replication numbers for the elements $1, \ldots, 9, A$ are as follows.

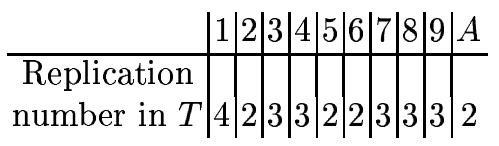

Assume $(1,2 ; 4) \in I_{1}$, where $I_{1}$ forms half of an interchange into which we are decomposing the partial latin square associated with $T$. Then by Lemma 3 we can say that 2 occurs only as a column and 4 occurs only as an element. Since 
$23 A$ is a triple, we have that $A$ occurs only as a row or an element, and since $49 A$ is a triple, we have that $A$ occurs only as a row or a column. Therefore, $A$ occurs only as a row and we must have $(A, 2 ; 3),(A, 9 ; 4) \in I_{1}$. Then we must have $(8,9 ; 3) \in I_{1}$. Since 678 is a triple, we have that 6 occurs only as a column or an element, and since 136 is a triple, we have that 6 occurs only as a row or a column. Therefore, 6 occurs only as a column and we must have $(8,6 ; 7),(1,6 ; 3) \in I_{1}$. However this leads to a contradiction since in the triple 457,4 and 7 must both be elements. Therefore no decomposition exists.

Trade of volume $9 \mathcal{T}_{21}=\left(T, T^{\prime}\right)$ where $T=\{123,145,167,189,24 A, 346,358$, $39 A, 479\}$ and $T^{\prime}=\{124,136,158,179,23 A, 345,389,467,49 A\}$.

The replication numbers for the elements $1, \ldots, 9, A$ are as follows.

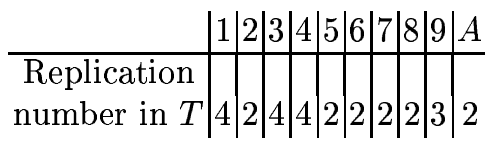

Assume that the partial latin interchange $I$ associated with $T$ can be decomposed into six disjoint latin interchanges, then since volume $(T)=9$ one of these latin interchanges, $\mathcal{I}_{1}=\left(I_{1}, I_{1}^{\prime}\right)$ say, must have type

$$
\left(\begin{array}{c}
W \\
X \\
Y
\end{array}\right)
$$

where $W, X$ and $Y$ are all odd and represent the appropriate sums for values of $\left|\mathcal{R}_{I_{1}}^{r}\right|,\left|\mathcal{C}_{I_{1}}^{c}\right|$ and $\left|\mathcal{E}_{I_{1}}^{e}\right|$. However it is not possible to partition the multiset $\{4,2,4,4,2,2,2,2,3,2\}$ into three multisubsets such that the sum of the entries in each of these multisubsets is odd. Therefore no such decomposition exists.

Trade of volume $9 \mathcal{T}_{22}=\left(T, T^{\prime}\right)$ where $T=\{123,145,167,189,24 A, 36 A, 468$, $479,578\}$ and $T^{\prime}=\{124,136,158,179,23 A, 457,46 A, 489,678\}$.

The replication numbers for the elements $1, \ldots, 9, A$ are as follows.

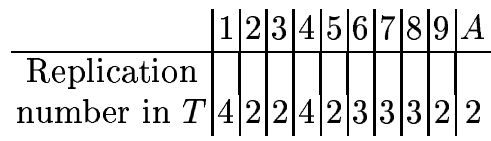

Assume $(5,7 ; 8) \in I_{1}$, where $I_{1}$ forms half of an interchange into which we are decomposing the partial latin square associated with $T$. Then by Lemma 3 we can say that 5 occurs only as a row, 7 occurs only as a column, and 8 occurs only as an element. On the other hand, since 167 and 468 are triples we must have $(6,7 ; 1),(6,4 ; 8) \in I_{1}$. Now considering the triples $36 A$ and 479 , we have four different cases:

Case $1\{(6,3 ; A),(4,7 ; 9)\} \subseteq I_{1}$ which means that 9 occurs only as an element by Lemma 3. But 189 as a triple requires both 8 and 9 to be elements. This is a contradiction. 
Case $2\{(6,3 ; A),(9,7 ; 4)\} \subseteq I_{1}$ which means that 9 occurs only as a row, A occurs only as an element, and 3 occurs only as a column by Lemma 3 . Then 189 as a triple requires that $(9,1 ; 8)$ be an element in $I_{1}$. Thus 1 occurs as both a column and an element. Since 123 is a triple, this means that $(2,3 ; 1)$ must be an element in $I_{1}$, which means that 2 can only occur as a row. Then $24 A$ as a triple requires $(2,4 ; A)$ to be an element of $I_{1}$. Thus 4 occurs as both a column and an element. Then 145 as a triple requires $(5,1 ; 4)$ to be an element of $I_{1}$, since column 1 needs to have two elements in it. It is now easy to see that $I_{1}$ with these entries cannot be a latin interchange. This is a contradiction.

Case $3\{(6, A ; 3),(4,7 ; 9)\} \subseteq I_{1}$ which means that 9 occurs only as an element by Lemma 3 , leading to a contradiction as in Case 1 .

Case $4\{(6, A ; 3),(9,7 ; 4)\} \subseteq I_{1}$ which means that 9 occurs only as a row, A occurs only as a column, and 3 occurs only as an element by Lemma 3 . Then 189 as a triple requires that $(9,1 ; 8)$ be an element in $I_{1}$. Thus 1 occurs as both a column and an element. Since 123 is a triple, this means that $(2,1 ; 3)$ must be an element in $I_{1}$, which means that 2 can only occur as a row. Then $24 \mathrm{~A}$ as a triple requires $(2, A ; 4)$ to be an element of $I_{1}$. Thus 4 occurs as both a column and an element. Then 145 as a triple requires $(5,4 ; 1)$ to be an element of $I_{1}$, since row 5 needs to have two elements in it. It is now easy to see that $I_{1}$ with these entries cannot be a latin interchange. This is a contradiction.

Thus no decomposition is possible.

Trade of volume $9 \mathcal{T}_{23}=\left(T, T^{\prime}\right)$ where $T=\{123,145,167,248,368,49 A, 579$, $69 B, 8 A B\}$ and $T^{\prime}=\{124,136,157,238,459,679,48 A, 68 B, 9 A B\}$. A decomposition exists where one interchange is given by $\mathcal{I}_{1}=\left(I_{1}, I_{1}^{\prime}\right)$ where $I_{1}=$ $\{(3,2 ; 1),(4,5 ; 1),(7,6 ; 1),(4,2 ; 8),(3,6 ; 8),(4, A ; 9),(7,5 ; 9),(B, 6 ; 9),(B, A ; 8)\}$.

Trade of volume $9 \mathcal{T}_{24}=\left(T, T^{\prime}\right)$ where $T=\{123,145,167,189,24 A, 36 A, 49 B$, $58 B, 79 A\}$ and $T^{\prime}=\{124,136,158,179,23 A, 45 B, 67 A, 89 B, 49 A\}$. A decomposition exists where one interchange is given by $\mathcal{I}_{1}=\left(I_{1}, I_{1}^{\prime}\right)$ where $I_{1}=$ $\{(3,2 ; 1),(4,5 ; 1),(7,6 ; 1),(8,9 ; 1),(4,2 ; A),(3,6 ; A),(4,9 ; B),(8,5 ; B),(7,9 ; A)\}$.

\section{References}

1. L. Brankovic, P. Horak, M. Miller and A. Rosa, Premature partial latin squares, Ars Combin. (to appear).

2. D. Donovan and A. Howse, Towards the spectrum of critical sets, Australas. J. Combin., 21, 2000, 107-130.

3. D. Donovan, A. Khodkar and A. P. Street, On doubling and tripling constructions for defining sets, (submitted).

4. D. Donovan, A. Khodkar and A. P. Street, On minimal defining sets in $A G(d, 3)$, (submitted).

5. A.D. Keedwell, Critical sets and critical partial latin squares, Proc. Third ChinaUSA Internation. Conf., Beijing, June 1993, World Scientific Publ. Co. Singapore, 1994, 111-124. 
6. G. B. Khosrovshahi and H. R. Maimani, On $2-(v, 3)$ Steiner trades of small volumes, Ars Combinatoria, 52, 1999, 199-220.

7. A. P. Street, Trades and defining sets, in CRC Handbook of Combinatorial Designs (edited C.J.Colbourn and J.H.Dinitz), CRC Publishing Co, 1996, pp 474478. 